\title{
REVISIÓN
}

\section{Pasado, presente y futuro de la citogenética en Costa Rica}

\author{
Isabel Castro Volio \\ Sección de Genética Humana, Instituto de Investigaciones en Salud, Universidad de Costa Rica. 2060 San José, Costa Rica. \\ Fax (506) 207 5130; icastro@cariari.ucr.ac.cr
}

Recibido 06-II-2004. C Corregido 24-VIII-2004. Aceptado 24-VIII-2004.

\begin{abstract}
Past, present and future of cytogenetics in Costa Rica. This is an overview of the past, present and future of human Cytogenetics in Costa Rica. It started in 1965 at the University of Costa Rica where it has been developed slowly but steadily. There is only one overloaded clinical cytogenetic laboratory in the social security system. The tests currently performed are peripheral blood and blood marrow karyotypes, prenatal cytogenetic diagnosis (amniotic fluid and fetal blood) and less frequently skin biopsies. The task now is to standardize molecular cytogenetic techniques, we are actually working with PRINS in order to study submicroscopic subtelomeric rearrangements associated with mental retardation and other microdeletion syndromes as well. Rev. Biol. Trop. 52(3): 537-544. Epub 2004 Dic 15.
\end{abstract}

Key words: Cytogenetics, karyotypes, human genetics, Costa Rica.

Palabras clave: Citogenética, cariotipos, genética humana, Costa Rica.

Las enfermedades genéticas en general, y los defectos cromosómicos en particular, son factores importantes como responsables de enfermedad, discapacidad y mortalidad humana. Su protagonismo aumenta conforme disminuye la morbi-mortalidad por causas de más fácil prevención, como las enfermedades infectocontagiosas y la desnutrición. Costa Rica no ha escapado a esta transición, y desde hace ya bastantes años, las enfermedades genéticas y los defectos congénitos, de más difícil prevención, son cada vez más importantes para la salud pública. En Costa Rica se han logrado importantes mejoras en los índices de salud, particularmente a partir de la década de los años 70, como consecuencia de profundos cambios políticos y socioeconómicos que han tenido efecto directo en las condiciones de vida y salud de la población. Se ha priorizado la atención primaria de salud y la atención del binomio madre-niño. Como resultado de estas acciones la mortalidad infantil disminuyó de 56.4 por mil nacidos vivos en 1971 a 15.3 en
1990 y 13.2 en 1995 (Anónimo 1996). Es conocido que de un 3\% a un 5\% de los niños nacidos en una población y período dados, presentan una anomalía congénita, que puede ser detectada al nacimiento. Sin embargo, la fracción que se manifiesta en recién nacidos es pequeña, después de un año de vida, su incidencia aumenta hasta un $10 \%$. Las malformaciones congénitas como causa de muerte en niños menores de un año (mortalidad infantil), han tenido un aumento relativo en los últimos años, ocupando el segundo lugar desde 1978. Según datos del Ministerio de Salud de Costa Rica, el porcentaje de la mortalidad por anomalías congénitas con respecto al total de defunciones infantiles, ha ido creciendo paulatinamente hasta alcanzar cifras de $32 \%$ para el año 1997 (Anónimo 1998). Los defectos cromosómicos tienen una cuota importante de responsabilidad en esta y otras patologías.

En el campo específico de las cromosomopatías, se distinguen dos grandes grupos: las constitucionales, con las que la persona 
nace, y las adquiridas, que surgen en algún momento después del nacimiento. Las primeras, se asocian a malformaciones congénitas de todo tipo y además el retardo mental es invariable, excepto para las aberraciones que afectan el número de cromosomas sexuales, en las cuales predominan los problemas de infertilidad. Las adquiridas son causa de gran variedad de cánceres. Las cromosomopatías constitucionales de cualquier tipo afectan a 1:156 recién nacidos, y 1:500 personas es portadora sana de algún tipo de rearreglo cromosómico balanceado, que no le ocasiona problema a ella misma, pero sí puede ser grave para su descendencia. El defecto cromosómico más frecuente en los recién nacidos es la trisomía 21 que origina el síndrome de Down, se presenta en 1:600 a 1:800 bebés. El síndrome de Down es la causa más común de retardo mental severo. El coeficiente intelectual promedio a la edad de 21 años es de alrededor de 42 , que equivale a una edad mental de 5 años. De los adultos de 21 años, únicamente el $40 \%$ se alimentan, visten, bañan y van al servicio sanitario sin ayuda y solamente alrededor del $17 \%$ se pueden dejar en casa desatendidos. Este síndrome también se asocia con problemas de salud importantes, alrededor del $50 \%$ de los recién nacidos requieren cirugía por al menos una anomalía congénita seria, usualmente cardiopatía (45\%) y defectos gastrointestinales (6\%). Además son más susceptibles a padecer leucemia, parálisis cerebral, hidrocefalia, hipotiroidismo, epilepsia y enfermedad de Alzheimer. La mortalidad asociada a esta trisomía es alta, 4 de cada 5 casos sobreviven a la edad de 5 años. Su expectativa de vida es de alrededor de 60 años. En vista de que la mayoría de las cromosomopatías, incluyendo la trisomía 21, obedecen a mutaciones nuevas o de novo, y tomando en cuenta que son de diagnóstico relativamente fácil, pero de tratamiento inexistente (a lo sumo rehabilitación), la prevención surge como una de las acciones de salud, en materia de genética, más importantes. Las herramientas con que contamos actualmente para lograr esta prevención son principalmente el asesoramiento genético, la identificación de grupos de mayor riesgo, el tamizaje poblacional en el suero de las embarazadas y el diagnóstico prenatal.

El desarrollo de la citogenética humana en Costa Rica se inició hace poco más de 40 años, en el Departamento de Anatomía Patológica de la recién abierta Facultad de Medicina de la Universidad de Costa Rica. El joven profesor Pedro Morera Villalobos había hecho recientemente una pasantía académica en la Universidad del Estado de Lousiana donde había entrado en contacto con esta ciencia que acababa de florecer y cuyo aroma embriagaba a muchos citopatológos en Europa y Norteamérica. Como don Pedro se formó en Italia, en la antiquísima Universidad de Pavia, era natural que quisiera combinar la docencia con la investigación, de manera que montó un pequeño laboratorio de citogenética y en una carta fechada 14 de junio de 1963 escribe “...Después de algún tiempo de entrenamiento, hemos finalmente logrado adquirir la técnica necesaria para este tipo de trabajo. Hasta ahora hemos limitado nuestra actividad a estudiar solamente material humano, de casos de mongolismo, hermafroditismo y leucemias monocíticas; es nuestra intención extender nuestro trabajo hacia el estudio de otras enfermedades. ..." Gracias a estos esfuerzos se produjo primero el matrimonio del director de tesis y la tesiaria y poco después la tesis de grado "Contribución al estudio de las anormalidades en los cromosomas humanos" de la estudiante de la Facultad de Microbiología Juanita Yock Apuy (Yock 1965). Gracias al regalo de don Pedro, conservo un ejemplar de este trabajo pionero, con las páginas amarillentas y fiel reflejo de los conocimientos que en ese momento se tenían de la nueva ciencia-arte de la citogenética y de las aberraciones cromosómicas y sus repercusiones fenotípicas. Muchos conceptos ya han sido dejados atrás, pero las fotos muestran cromosomas con tinción uniforme - no se habían inventado las bandas - de muy buena morfología y clasificados según la propuesta del grupo de Denver.

Se produjo una brecha de 10 años en la cual el país se quedó rezagado. En 1975, a instancias de nuestro primer genetista clínico nacional, 
Mario Saborío Ruíz, se le encomienda al joven microbiólogo Julio Rivera, en ese entonces encargado de las serologías, la labor de montar el laboratorio de citogenética del Hospital Nacional de Niños Carlos Sáenz Herrera. Durante el año 1978 Julio realizó una pasantía en México en el Instituto de la Nutrición, bajo la tutela de los afamados citogenetistas Rubén Lisker y Osvaldo Mutchinic. Para cultivar los linfocitos y obtener las preparaciones cromosómicas, Julio ya no abría la refrigeradora y tomaba los reactivos listos para ser usados, como en México, sino que debía ir al matadero de Montecillos a buscar el suero bovino fetal para luego procesarlo. Debía además sembrar y cosechar los frijoles que le regalaban en la Facultad de Agronomía, licuarlos y hacer diluciones cada vez que necesitaba fitohemaglutinina. También tenía que ir al Hospital México a conseguir pastillas de colchicina, que luego molía y diluía y además periódicamente debía tomar el autobús para desplazarse hasta INCIENSA donde filtraba los medios de cultivo. Y hasta el día de hoy Julio sigue a cargo de ese laboratorio, el único que presta servicios a todos los pacientes atendidos en la Caja Costarricense del Seguro Social, sin perder esa enorme mística de trabajo y con muy poco personal de apoyo. Además es profesor colaborador del curso de Genética Médica de la Escuela de Medicina de la Universidad de Costa Rica desde hace muchos años. A pesar de que el volumen de trabajo es asfixiante, Julio y su pupila, Patricia Venegas, han conseguido liberarse de la rutina cada vez que han podido y han hecho publicaciones interesantes (Rivera et al. 1981, 1984, Sánchez et al. 1995, Sánchez y Venegas 1996, Salazar et al. 1997, Venegas et al. 1997, Venegas y Rivera 2004).

En 1979 se inició la citogenética en el Instituto de Investigaciones en Salud, pero no dentro del campus de la Universidad de Costa Rica, pues el INISA aún no tenía edificio propio, sino en el hospital R. A. Calderón Guardia, con Virginia Solís Alvarado, que venía de la Universidad de Bucarest, en Rumania, con un doctorado en biología, con énfasis en citogenética humana y experimental (Solís 1977a, Solís 1977b, Solís 1978). En 1982 se sumó al INISA Patricia Cuenca para realizar su tesis de maestría en biología (Cuenca y Arias 1985, Cuenca 1986), bajo la dirección de Virginia. Esta última, en 1984 dejó el INISA para pasar a la Escuela de Biología de la Universidad de Costa Rica, donde es profesora hasta la fecha y ha seguido publicando, sobre todo en citogenética de tumores (Solís 1982, 1983, 1984, 1985, 2001, Solís et al. 1988, 2000).

Por otra parte, en INCIENSA, la bióloga Marta Cruz y la microbióloga María Cecilia Matamoros también hicieron cariotipos posnatales durante algún tiempo, estudiaron el síndrome de Down y también sitios frágiles cromosómicos en una población con esquizofrenia (Cruz et al. 1983, Handal et al. 1988).

En 1984 la autora de este artículo, médico citogenetista formada en el Hospital Karolinska en Suecia, fue contratada en el momento en que se trasladaba el laboratorio de citogenética del hospital al nuevo edificio del INISA, ubicado en lo que ahora se llama la Ciudad de la Investigación de la Universidad de Costa Rica. De esta manera, a pesar de las posiciones antagónicas de varios médicos, se inició el diagnóstico fetal citogenético en nuestro país (Castro y Jiménez 1985, Castro et al. 1995, 2000, 2001, Castro y Ortiz 2003, Castro Volio 2004). Al mismo tiempo, junto con Patricia Cuenca, se estudió una población con retardo mental de la principal escuela de enseñanza especial del país, donde detectamos el primer caso nacional del síndrome del cromosoma $\mathrm{X}$ frágil, el cual es la causa más frecuente de retardo mental hereditario en el mundo, pero cuyo diagnóstico nunca antes se había hecho en nuestro medio (Castro y Cuenca 1987, 1996). Desde entonces no se ha dejado de investigar en este campo, Patricia además ha estandarizado el diagnóstico molecular de ésta y de otras patologías causadas por mutaciones inestables (Cuenca y Morales 2004) de manera que el estudio citogenético no se hace más por ser menos confiable y más caro.

En 1988 Patricia Cuenca obtuvo una beca para hacer el doctorado en el Instituto de Biología 
Humana de la Universidad de Hamburgo y regresó al INISA luego de presentar su tesis acerca de polimorfismos cromosómicos en diferentes grupos étnicos de Costa Rica (Cuenca 1992). Ha hecho estudios en toxicología genética, algunos de los cuales incluyen aberraciones cromosómicas en linfocitos de personas expuestas a mutágenos ambientales (Cuenca et al. 1997, Castro et al. 2004).

Hace poco más de diez años en el INISA, junto con el Hospital México, se comenzó a hacer estudios de cromosomopatías adquiridas por 117 enfermos adolescentes y adultos con leucemias, con síndromes mieloproliferativos, mielodisplásicos e hipereosinofílicos (Castro et al. 1993). Desde entonces, y especialmente ahora que existe la posibilidad de tratar a los pacientes con leucemia mieloide crónica con un medicamento específico para esta enfermedad, se hace diagnóstico de cromosoma Filadelfia para confirmar el diagnóstico clínico y citopatológico inicial. Este servicio se ofrece a todos los hospitales de la seguridad social y a hospitales privados.

En otro tipo de estudio realizado en el INI$\mathrm{SA}$, se determinaron las consecuencias citogenéticas de la infección con el virus de inmunodeficiencia humana (VIH), mediante un estudio tipo doble-ciego de casos infectados o VIH+ $(\mathrm{N}=17)$ y controles VIH - $(\mathrm{N}=17)$, en 34 hombres de una población muy homogénea de homosexuales, estudiada de 1988 a 1991. Se encontró fragilidad cromosómica no diferente del porcentaje informado para la población general ni diferente entre casos y controles $(\mathrm{O} . \mathrm{R} .=1.18)$. Las diferencias entre ambos grupos fueron más bien cualitativas, en los sitios de lesión cromosómica, ya que los hombres infectados mostraron cuatro veces más lesiones en dos sitios en particular: $2 \mathrm{p} 21$ y $3 \mathrm{p} 21$. Este último coincidió con el sitio de fragilidad más común informado con anterioridad. Este sitio en 3 p21 fue posteriormente identificado como el locus cromosómico donde se ubica el gen CKR5 el cual codifica para un receptor secundario en el linfocito $\mathrm{T}$ del VIH-1. Se propuso que este gen se estaba transcribiendo activamente en los hombres infectados en los que se evidenciaron lagunas o fracturas cromosómicas en 3p21 (Castro-Volio y Valle-Bourrette 2002).

Además de los cariotipos fetales y estudios cromosómicos en onco-hematología, en el INISA hacemos también cariotipos pos-natales en recién nacidos con fenotipos sugerentes de cromosomopatía, en niños con problemas de retardo mental y en parejas con historia médica de abortos a repetición o de infertilidad. Se tiene un trabajo que investiga por primera vez en Costa Rica la causalidad de los defectos cromosómicos en los problemas de infertilidad y aborto habitual. Se estudiaron 71 parejas mediante análisis citogenético según técnicas usuales. Las parejas en las cuales se detectó cromosomopatía recibieron asesoramiento genético. De las 142 personas estudiadas, todas menos siete tuvieron el cariotipo normal. Esto representa una frecuencia de $4.9 \%$ de cromosomopatía en esta población. En el SOREP (Centro Universitario de Investigación en Poblaciones) de la Universidad de Quebec en Canadá se instaló una base de datos computarizada generada a partir de la literatura referente a estudios citogenéticos en parejas con pérdida gestacional recurrente. Hasta 1990 contenía información de 22199 parejas, de las cuales, el $4.7 \%$ incluía un miembro portador de una anomalía cromosómica. Esta cifra es muy similar a la encontrada por nosotros en este estudio (Castro y Ortiz 2002). El asesoramiento genético y el diagnóstico prenatal son acciones de salud necesarias y adecuadas para prevenir el nacimiento de productos con defectos cromosómicos en estas parejas.

A pesar de los avances en materia de atención de la salud en nuestra población, persiste el problema de acceso inadecuado y demanda aumentada de servicios de diagnóstico citogenético. En el ámbito de la seguridad social la capacidad del único laboratorio es muy reducida y los tiempos de espera son largos. Por otro lado, la mayoría de los médicos no tienen claras las indicaciones para solicitar el cariotipo. Se debe estudiar la presencia de cromosomopatía en todos los casos anotados en el cuadro 1. 
CUADRO 1

Indicaciones para realizar el cariotipo posnatal

$\begin{array}{ll}\text { Grupo etario } & \text { Indicaciones } \\ \text { Neonatal } & \text { Rasgos dismórficos } \\ & \text { Anomalías congénitas } \\ & \text { Ambigüedad sexual } \\ \text { Pediátrico } & \text { Rasgos dismórficos } \\ & \text { Discapacidad física } \\ & \text { Retardo psicomotor con o sin problemas } \\ & \text { de lenguaje } \\ & \text { Retardo mental } \\ & \text { Leucemia } \\ \text { Adolescente } & \text { Retraso puberal } \\ & \text { Amenorrea } \\ & \text { Estatura muy alta o muy baja } \\ & \text { Retraso en el desarrollo } \\ & \text { Leucemia } \\ & \text { Dos o más abortos espontáneos } \\ \text { Adultos } & \text { Infertilidad } \\ & \text { Mortinatos } \\ & \text { Leucemia } \\ & \text { Linfoma }\end{array}$

A diferencia de la mayoría de los tumores, que afectan primordialmente a la población de mayor edad, las hemopatías malignas se ensañan con los niños, adolescentes y con los adultos en las etapas de la vida más productivas. Causan por lo tanto, y debido a su alta mortalidad, mucho sufrimiento a los pacientes y a sus familias, además de una debacle económica para las mismas. Esta situación ha generado mucha investigación biomédica y grandes adelantos terapéuticos que han contribuido a prolongar la vida de los pacientes e incluso en muchos casos a curarlos. Estos adelantos que actualmente son empleados de rutina en muchos países, incluso en aquellos con desarrollo similar al nuestro, no se han visto reflejados en el manejo de nuestros pacientes, particularmente en lo referente a la utilización de las aplicaciones clínicas de las nuevas técnicas de citogenética molecular. $\mathrm{Mu}$ chos de nuestros enfermos adultos corren el riesgo de estar mal clasificados por falta de la información citogenética pertinente, la mayoría de ellos no se pueden estratificar y tratar de acuerdo al riesgo que les confiere determinada alteración cromosómica, muchos tampoco se pueden monitorear adecuadamente para valorar la respuesta al tratamiento y anticipar las recaídas de la enfermedad. Desconocemos además cómo se comportan la mayoría de los enfermos adultos costarricenses desde el punto de vista genético. Con el fin de subsanar estas deficiencias se está buscando apoyo económico para desarrollar un proyecto con el objetivo de caracterizar mediante citogenética convencional y molecular los pacientes con cuadro clínico de leucemia. Esto permite clasificar los pacientes adecuadamente, estimar el riesgo que corren, seleccionar el mejor tratamiento con base en ese diagnóstico y ese pronóstico, valorar la respuesta al mismo y monitorear al paciente durante cinco años para anticipar el riesgo de recaída. De esta manera es posible propiciar el cuidado más adecuado de los pacientes con leucemia o síndromes mielodisplásicos para conseguir la curación completa o al menos prolongar sus vidas libres de enfermedad.

Otro proyecto que se va a iniciar a corto plazo es el estudio de personas con retardo mental idiopático para buscar alteraciones subteloméricas. Informes recientes responsabilizan a los reacomodos subteloméricos cromosómicos como la segunda causa genética de deficiencia mental. Muchos genes relacionados con el cerebro se ubican cerca del extremo de los cromosomas, junto a secuencias de $\mathrm{ADN}$ que protegen las puntas cromosómicas llamadas telómeros. Los telómeros son los extremos físicos de los cromosomas y funcionan como estuches protectores, consisten de una repetición simple de seis nucleótidos (TTAGGG) que es idéntica en cada cromosoma. Adyacente al telómero hay una segunda región de secuencias repetidas llamada "repeticiones subteloméricas" o también "repeticiones asociadas al telómero". A la par de éstas, como a $100 \mathrm{~kb}$ del extremo del cromosoma, empiezan las secuencias codificantes únicas. Toda esta región distal puede ser llamada "banda telomérica". Estas secuencias codificantes distales son ricas en genes y parece ser que cerca de un 5 a $6 \%$ de los casos de retardo mental de origen desconocido se relacionan con deleciones o translocaciones desbalanceadas en esos genes (Knight y Flint 2000). Los cariotipos de resolución estándar buscan cambios en 
el número y en la estructura normal de los cromosomas. Los estudios de alta resolución hacen posible detectar defectos cromosómicos poco evidentes. Pero esta tecnología resulta insuficiente para identificar los reacomodos subteloméricos y las microdeleciones que involucran menos de 2-3 megabases. En estos casos, la hibridación in situ fluorescente (FISH) permite evaluar la presencia de los defectos cromosómicos más pequeños. La técnica FISH utiliza segmentos fluorescentes de ADN como sondas genéticas que se unen a sus análogos en el ADN del propio paciente. Utilizando un microscopio de fluorescencia, el ADN de un paciente que tiene dos copias normales del segmento probado muestra dos puntos luminosos. Cuando uno de los segmentos de ADN que se está investigando está ausente o dañado, la sonda correspondiente no se hibrida y no fluoresce. Un inconveniente de esta técnica es que es laboriosa y cara si se compara con otra técnica menos usada llamada PRINS. Esta es una técnica rápida y sensible, alternativa a las tradicionales metodologías de FISH. La estrategia consiste en que una sonda no marcada es mezclada con nucleótidos (de los cuales al menos uno está marcado con un hapteno) y polimerasa de ADN. Esta mezcla se aplica luego a una preparación de células en metafase o en interfase. Cuando la sonda hibrida con su secuencia diana en la cromatina, funciona como un iniciador para la elongación de la cadena usando el ADN cromosómico como molde. La elongación de la cadena es catalizada por la polimerasa del $\mathrm{ADN}$, que incorpora los nucleótidos y por lo tanto marca el sitio de la hibridación (Bonifacio et al. 2001). Se van a probar estas técnicas y utilizar la que mejores resultados proporcione y al menor costo, con el objeto de detectar alteraciones subteloméricas en personas con retardo mental idiopático. Esto nos va a permitir asesorar a las familias en las que se presentan casos de mutaciones cromosómicas subteloméricas submicroscópicas, con las siguientes metas:
- La prevención de la presencia o recurrencia de retardo mental en las familias en las que segregan mutaciones subteloméricas.

- La posibilidad de que en las familias con mutaciones de novo se recupere la confianza en cuanto a la posibilidad de procrear hijos de inteligencia normal.

- La obtención del diagnóstico definitivo que permite el cese de la búsqueda del origen del retardo mental en la persona afectada. Esto implica una serie de múltiples estudios de laboratorio y de radioimágenes que son muy onerosos para las familias y para la seguridad social.

Realmente es muy amplio el campo de la investigación básica en citogenética, pero en el INISA hemos preferido dedicarnos al tipo de investigación aplicada dado que en el país existen necesidades básicas de diagnóstico citogenético que no son cubiertas sino mínimamente por los servicios de salud. Junto con la Escuela de Biología hemos formado suficientes biólogos no solamente en citogenética médica sino en genética humana en general, que podrían prestar excelentes y muy necesarios servicios a la salud pública del país en los hospitales estatales, pero que por el momento no han tenido la oportunidad de trascender más allá de las universidades por entrabamientos administrativos y gremiales.

\section{RESUMEN}

Esta es una breve descripción de lo que ha sido el desarrollo de la citogenética humana en Costa Rica, la situación actual y las expectativas a corto plazo. Esta ciencia-arte nació hace poco más de cuarenta años en la Facultad de Medicina de la Universidad de Costa Rica. Actualmente se desarrolla como un servicio diagnósticoasistencial en el Hospital Nacional de Niños "Dr. Carlos Sáenz Herrera" y con un componente adicional de investigación en el Instituto de Investigaciones en Salud de la misma universidad. Los ensayos que se ofrecen a la población son los cariotipos en sangre periférica y en médula ósea, el diagnóstico prenatal citogenético en 
muestras de líquido amniótico y de sangre fetal y con menos frecuencia en biopsias de piel.

\section{REFERENCIAS}

Anónimo. 1996. Análisis de la situación de la salud, documento preliminar, Mortalidad infantil por grupo de causas. Costa Rica, Período 1970-1994. INCIEN$\mathrm{SA} / \mathrm{MS}$.

Anónimo. 1998. Porcentaje de la mortalidad por anomalías congénitas con respecto al total de defunciones infantiles, Costa Rica 1990-1997. Dirección General de Estadística y Censos y Ministerio de Salud, Departamento Centro de Información, Sección Otros Programas Prioritarios.

Bonifacio, S., C. Centrone, L. Da Prato, M.R. Scordo, M. Estienne \& F. Torricelli. 2001. Use of primed in situ labelling (PRINS) for the detection of telomeric deletions associated with mental retardation. Cytogenet. Cell Genet. 93:16-18.

Castro, R., V. Ramírez \& P. Cuenca. 2004. Análisis de micronúcleos y otras anormalidades nucleares en el epitelio oral de mujeres expuestas ocupacionalmente a plaguicidas. Rev. Biol. Trop. 52(3): 207-217.

Castro, I. \& J. Jiménez. 1985. Diagnóstico prenatal de aberraciones cromosómicas en Costa Rica. Rev. Méd. Hosp. Nal. Niños (Costa Rica). 20: 111-120.

Castro, I. \& P. Cuenca. 1987. Tamizaje de sitio frágil en el cromosoma X en una población de retardados mentales. Hallazgos preliminares. Rev. Méd. Hosp. Nal. Niños (Costa Rica). 1: 11-14.

Castro, I., C. Montero \& G. Jiménez. 1993. Características cromosómicas asociadas con leucemias y otras hematopatías en Costa Rica. Rev. Biol. Trop. 41: 385 392.

Castro, I., G. Escalante, H. Mora, D. Guerra, L. Sánchez \& C. Peña. 1995. Cariotipo de células fetales en el diagnóstico prenatal en Costa Rica. Rev. Biol. Trop. 43: 31-37.

Castro Volio, I. \& P. Cuenca Berger, 1996. Frecuencia del síndrome del cromosoma $\mathrm{X}$ frágil en la Escuela de Enseñanza Especial "Fernando Centeno Güell". Acta Ped. Cost. 10: 99-106.

Castro Volio, I., K. Sander Mangel, M. Vargas Prado, L. Sánchez Cháves \& G. Escalante López. 2000. Cariotipos fetales en embarazos de alto riesgo genético provenientes de hospitales de la seguridad social y de la consulta privada, de 1993 a 1998. Acta Méd. Cost. 42: $25-30$.
Castro Volio, I., Sander Mangel K., Vargas Prado M., Sánchez Cháves L. y Escalante López G. 2001. Diagnóstico prenatal citogenético mediante amniocentesis durante los trimestres II y III de gestación en Costa Rica. Rev. Biol. Trop. 49: 1227-1236.

Castro-Volio, I. \& L. Valle-Bourrette. 2002. Chromosomal defects in 34 male homosexuals, half of them with HIV antibodies. Rev. Biol. Trop. 50(1): 347-353.

Castro Volio, I. \& F. Ortiz Morales. 2002. Cariotipos conyugales en infertilidad y pérdidas gestacionales en Costa Rica. Toko-Gin Prac. 61: 332-336.

Castro Volio, I. \& F. Ortiz Morales. 2003. Cordocentesis para diagnóstico fetal citogenético en Costa Rica. Progr. Diag. Trat. Prenat. 15: 116-119.

Castro Volio, I. 2004. El diagnóstico prenatal de defectos cromosómicos en Costa Rica. Rev. Biol. Trop. 52(3): 141-146.

Cruz, M., A.E. Giovannetti, C. De Céspedes \& R. Fernández. 1983. Relación entre síndrome de Down, edad materna y presencia de translocaciones cromosómicas. Acta Méd. Cost. 26: 54.

Cuenca, P. \& E. Arias. 1985. Malformaciones congénitas en el Hospital Calderón Guardia. Frecuencia y estudio citogenético. Rev. Med. Hosp. Nal. Niños (Costa Rica). 20: 97-110.

Cuenca P. 1986. Recién nacidos con malformaciones congénitas, citogenética y aspectos epidemiológicos. Hospital Dr. Rafael Angel Calderón Guardia, 19821983. Tesis de maestría. Sistema de Estudios de Posgrado, Universidad de Costa Rica, Costa Rica. 134 p.

Cuenca, P. 1992. Untersuchung chromosomaler heteromorphismen bei unterschiedlichen ethnischen Gruppen Costa Ricas und ihre Bedeutung fur die Populationsgenetik. Doktorarbeit. Fachbereich Biologie and Universitat Hamburg, 157 p.

Cuenca, P., V. Ramírez, R. Castro \& K. Schosínsky. 1997. Efectos genotóxicos de los pesticidas en una población costarricense con exposición ocupacional. Micronúcleos de linfocitos y mucosa oral, aberraciones cromosómicas, mecanismos de reparación y electroforesis de células únicas, controlando paralelamente los niveles de colinesterasa sérica y eritrocítica. Informe final ante PLAGSALUD/OPS. 62 p.

Cuenca, P. \& F. Morales. 2004. Las mutaciones inestables, nuevo reto para el consejo genético de enfermedades hereditarias. Rev. Biol. Trop. 52(3): 87-95.

Handal, N. , M.C. Matamoros, M.E. Cruz \& R. Barrantes. 1988. Estudio de sitios frágiles cromosómicos en una 
población de esquizofrénicos de Cartago, Costa Rica. Acta Méd. Cost. 31: 103-107.

Knight, S. J. \& J. Flint. 2000. Perfect endings: a review of subtelomeric probes and their use in clinical diagnosis. J. Med. Genet. 37: 401-409.

Rivera, J., F. Cruz, M. Saborío \& O. Sesín. 1981. Trisomía 18. Rev. Med. Hosp. Nal. Niños (Costa Rica). 16: $97-$ 102.

Rivera, J., M. Saborío \& F. Cruz. 1984. Un síndrome cromosómico poco frecuente: trisomía 22. Rev. Med. Hosp. Nal. Niños (Costa Rica). 19: 19-24.

Salazar, L., L.F. Vázquez, J. Rivera \& P. Venegas. 1997. Estudio citogenético en médula ósea de 26 casos de leucemia mieloide crónica en pacientes del Hospital San Juan de Dios. Rev. Cost. Cienc. Méd. 18: 44-48.

Sánchez, M.A., P. Venegas \& J. Rivera. 1995. Hallazgo de una deleción en el brazo largo del cromosoma 22 en un paciente con problemas de lenguaje. Rev. Med. Hosp. Nal. Niños (Costa Rica). 30: 5-8.

Sánchez, M.A. \& P. Venegas. 1996. Inversión paracéntrica en el brazo corto del cromosoma 1, en un paciente con problemas de obesidad y aprendizaje. Rev. Med. Hosp. Nal. Niños (Costa Rica). 31: 5-8.

Solís V. 1977a. Aspectos genéticos en la patología de las leucemias. Monografía. Universidad de Bucarest, Bucarest. 69 p.

Solís V. 1977b. Métodos de bandeo y su aplicación en el estudio de las aberraciones cromosómicas. Monografía. Universidad de Bucarest, Bucarest. 51 p.

Solís, V. 1978. Estudio comparativo de las modificaciones cromosómicas en diferentes tipos de proliferaciones malignas (humanas y experimentales). Tesis de doctorado. Universidad de Bucarest, Bucarest, Rumanía.

Solís, V. 1982. Estudio citogenético del tumor ascítico Guerin T8 después de diez años de pasajes en serie, utilizando hospederos homólogos. Acta Méd. Cost. 25: $125-134$.

Solís, V. 1983. Reaparición de una translocación no común implicada en la formación del cromosoma Philadelphia $\left(\mathrm{Ph}^{1}\right)$. Acta Méd. Cost. 26: 28-31.

Solís, V. 1984. Estudio citogenético de un caso de leucemia crónica linfocítica de células T. Acta Méd. Cost. 27: $170-175$.

Solís, V. 1985. Patrón de las asociaciones satelíticas en mujeres post-parto y en neonatos. Rev. Biol. Trop. 33: $51-56$.

Solís, V., J. Pritchard \& J. Cowell. 1988. Cytogenetic changes in Willms' tumours. Canc. Genet. Cytogenet. 34: 223-234.

Solís, V., M. de los A. Alvarado, E. Ruiz, J. Carrillo, M. Navarrete, G. Sánchez \& E. Jiménez. 2000. Citogenética y citoquímica de pacientes con leucemia en dos hospitales neotropicales. Rev. Biol. Trop. 48: 707-718.

Solís, V. 2001. Bandeo de cromosomas humanos utilizando extracto crudo de frutas u hojas de papaya. Rev. Biol. Trop. 49: 1127-1129.

Venegas, P. \& J. Rivera. 2004. Estudios citogenéticos en niños con leucemia linfocitica aguda-B en Costa Rica. Rev. Biol. Trop. 52(3): 147-154.

Venegas, P., A.V. Fallas, J. Rivera \& M.A. Sánchez. 1997. Frecuencia del síndrome del X frágil en la consulta de Genética Médica del Hospital Nacional de Niños. Rev. Méd. Hosp. Nal. Niños (Costa Rica). 32: 9-12.

Venegas, P., M.A. Sánchez \& J. Rivera. 1997. Hallazgo de una translocación 1;16 en una paciente con aparente fenotipo de síndrome de Down. Acta Ped. Cost. 11: 45-46.

Yock A., J. 1965. Contribución al estudio de las anormalidades de los cromosomas humanos. Tesis de grado. Universidad de Costa Rica, San José, Costa Rica. 41 p. 